\title{
Rotation and Spin and Position Operators in Relativistic Gravity and Quantum Electrodynamics
}

\author{
Robert F. O'Connell \\ Department of Physics and Astronomy, Louisiana State University, Baton Rouge, LA 70803-4001, USA; \\ oconnell@phys.lsu.edu; Tel.: +1-225-578-6848
}

Received: 3 December 2019; Accepted: 16 January 2020; Published: 26 January 2020

\begin{abstract}
First, we examine how spin is treated in special relativity and the necessity of introducing spin supplementary conditions (SSC) and how they are related to the choice of a center-of-mass of a spinning particle. Next, we discuss quantum electrodynamics and the Foldy-Wouthuysen transformation which we note is a position operator identical to the Pryce-Newton-Wigner position operator. The classical version of the operators are shown to be essential for the treatment of classical relativistic particles in general relativity, of special interest being the case of binary systems (black holes/neutron stars) which emit gravitational radiation.
\end{abstract}

Keywords: rotation; spin; position operators

\section{Introduction}

Rotation effects in relativistic systems involve many new concepts not needed in non-relativistic classical physics. Some of these are quantum mechanical (where the emphasis is on 'spin'). Thus, our emphasis will be on quantum electrodynamics (QED) and both special and general relativity. Also, as in [1], we often use 'spin' in the generic sense of meaning 'internal' spin in the case of an elementary particle and 'rotation' in the case of a macroscopic body.

In Section 2, we examine how spin is treated in special relativity by discussing spin supplementary conditions (SSC) and how it is related to the choice of a center-of-mass of a spinning particle. In Section 3, we discuss QED, particularly the Dirac equation and the Foldy-Wouthuysen transformation, which is used to consider positive energy particles. Even though it is not usually referenced to as a position operator, it is in fact equivalent to the Pryce-Newton-Wigner position operators, which we also discuss in detail in Section 3. In addition, the classical version of these operators turn out to be essential in treating classical relativistic particles in general relativity, which is the subject of Section 4. Of special interest is the treatment of spin precession in binary systems as well as gravitational radiation from the merger of the two objects (black holes or neutron starts). In Section 5 we discuss our results.

\section{Special Relativity}

In classical mechanics, the spin is denoted by the 3-vector $\vec{S}$. However, in special relativity, Mathisson [2], who pioneered the study of spin in general relativity, generalized this to an anti-symmetric second rank tensor $S_{\propto \beta}=-S_{\beta \alpha}$.

Another possibility is to define an axial 4-vector $S_{\propto}$ which reduces to the 3-vector $\vec{S}$ in the rest-frame of the particle:

$$
S_{\propto}=\frac{1}{2} \epsilon_{\propto \beta \sigma \tau} \quad S^{\beta \sigma} U^{\tau},
$$


where $\epsilon_{\propto \beta \sigma \tau \tau}$ is the completely antisymmetic Levy-Civita tensor, $U^{\tau}=(\gamma, \vec{v} / \mathrm{c})$ is the familiar 4-velocity, and $S^{\propto}=(0, \vec{S})$ in the rest frame where $\vec{v}=0$. Hence, using the fact that $\epsilon_{\alpha \beta \sigma \tau}$ is antisymmetric in $\propto$ and $\tau$, we obtain:

$$
U^{\propto} S_{\propto}=0,
$$

so that the 4-vectors $U^{\propto}$ and $S_{\propto}$ are not only orthogonal in the rest frame as constructed but are also orthogonal in all frames. In addition, using the properties of the Levi-Citiva symbol and $U^{\propto} U_{\propto}=-1$, (1) may be inverted to give:

$$
S^{\propto \beta}=\epsilon^{\propto \beta \sigma \tau} S_{\sigma} U_{\tau} .
$$

Equation (2) is a spin supplementary condition (SSC) which ensures that even when $\vec{v}$ is non-zero, $S^{\mu}$ has only three independent components and similarly for $S^{\propto \beta}$. In fact, we can obtain a different SSC by replacing $U^{\tau}$ by $P^{\tau}$, the 4 -momentum. This is related to the fact that there are essentially two basic rest systems for the particle, corresponding to either $\vec{v}=0$ or $\vec{p}=0$. As a result, the definition of a rest frame is related to a choice of SSC which, in turn, is related to the choice of a center-or-mass for the spinning particle [3]. Thus, switching from one SSC (or rest-frame) to another is exactly the same as shifting the center of mass by a Lorentz transformation.

\section{Quantum Electrodynamics (QED)}

A relativistic theory of the electron in the quantum regime is the Dirac equation. This equation reduces to the Pauli equation in the non-relativistic limit but in order to obtain in QED the various orders of relativistic corrections, written as terms involving powers of $1 / \mathrm{m}$ where $\mathrm{m}$ is the electron mass, the Dirac equation must be subjected to a unitary transformation, as written by Foldy-Wouthuysen (FW) in a pioneering paper [4]. In fact, the purpose of the FW transformation is to find a representation in which the small and large components are decoupled, so that one reduces to the Pauli description in the non-relativistic limit and the other describes the negative energy states. For this reason, the FW method is overwhelmingly used in discussing problems involving positive energy states $[5,6]$. The FW approach resulted in new position and spin operators [5,6] and in a famous footnote (number 7 of the manuscript), FW referred to two other significant papers, Pryce [7] and Newton-Wigner [8], the main goals of which were to obtain position operators. Pryce explored many possibilities for generalizing the Newtonian definition of the mass-center of a spinning body to special relativity and the preferred choice is $[7,8]$ :

$$
\Delta r=\frac{S \times P}{m\left(m c^{2}+P_{0} c\right)}
$$

where $\Delta r$ is the shift in the usual canonical orbital coordinate, $\mathrm{c}$ is the velocity of light and $S$ denotes the total spin.

Also:

$$
\mathrm{P}_{0}^{2} c^{2}=\mathrm{P}^{2} c^{2}+\mathrm{m}^{2} c^{4}=\left(\mathrm{P}_{1}^{2}+\mathrm{P}_{2}^{2}+\mathrm{P}_{3}^{2}\right) c^{2}+\mathrm{m}^{2} c^{4} .
$$

It turns out that the Pryce-Newton-Wigner (PNW) expression given in (4) is the same as the so-called mean position operator obtained by FW. The key goal of Newton-Wigner (NW) is to investigate the properties of a localized state for elementary systems of non-zero mass and arbitrary spin. The function they obtain has an associated position operator which has the property of preserving the positive energy of the wave function to which it is applied. In other words, NW are considering only positive energy particles in contrast to Dirac who considered both positive and negative energy particles. Moreover, NW also considered arbitrary spin of the constituent particles. They also required that the position operators commute with each other in addition to other invariance requirements. Especially important, as we shall discuss in more detail below, is the localization requirement. In 
particular, for the case of spin $0, \mathrm{NW}$ concluded that the only state which is localized at the origin at time $t=0$ is given in momentum space by:

$$
\psi_{0}(P)=(2 \pi)^{-\frac{3}{2}} P_{0}
$$

The corresponding localized wave function in coordinate space is such that it is spread out over a Compton wavelength [8].

In addition, the correct Hermitian position operator $\mathrm{q}^{\mathrm{k}}$ for the $\mathrm{k}$ - coordinate with an eigenvalue $\mathrm{x}^{\mathrm{k}}$ is calculated to be given by the equation:

$$
\mathrm{q}^{\mathrm{k}} \psi_{0}(\mathrm{p})=-\mathrm{i}\left(\partial / \partial \mathrm{P}_{k}+\mathrm{p}^{\mathrm{k}} / 2 \mathrm{P}_{0}^{2}\right) \psi_{0}(\mathrm{p})
$$

where only the first term in Equation (7) corresponds to the customary $\mathrm{q}^{\mathrm{k}}$ operator. As noted by Equation [8], this result for $\mathrm{q}^{\mathrm{k}}$ corresponds to the result given by Pryce [7] in for the case of spin $s=$ $1 / 2$. Pryce investigated how the mass-center of the constituent interacting particles could be defined in relativity and, in particular, he examined various generalizations of the Newtonian definition. He eventually settled on a definition which is the mean of the coordinates of the individual particles weighted with the total energy and the rest mass of the total system. This definition of the mass-center ensures that it is at rest in a frame in which the total momentum is zero but is does depend on the frame in which it is defined. In other words, it is not covariant but it does coincide with the choice of NW. It is written down explicitly in [4]. The equivalence of the Pryce and NW position operators for arbitrary spin has been given by Lorente and Roman [9], who made extensive use of gauge symmetries associated with the inhomogeneous Lorentz group generators.

In essence, Equation (4) was also used in the theoretical calculation of the 2-body spin precession which has now been verified by observations [10]. A notable derivation of (4) was carried out by Hanson and Regge [11] using a canonical formalism following Dirac's approach for constrained Hamiltonian systems [12]. This was followed up by a paper by Hojman and Regge [13] who used some improved techniques to obtain similar results and also generalize the previous special relativistic work to the framework of general Relativity. A similar approach to the problem is the work of Ramond [14].

Finally, we note that the NW operator $\mathrm{q}(\mathrm{t})$ obeys the relationship [15]:

$$
\langle\mathrm{q}(\mathrm{t})\rangle=\langle\mathrm{q}(0)\rangle+t\langle\mathrm{v}\rangle,
$$

where:

$$
\langle\mathrm{v}\rangle=\left(P / P_{0}\right) .
$$

Thus, the familiar relation between velocity and momentum in relativistic classical mechanics holds for the quantum mechanical operators of NW theory, which is a welcome property in applications.

\section{Classical Relativistic Systems}

Applications in QED did not refer to position operators per se and no thought was given to the possibility that they had an important role to play in classical relativistic systems, as was first discovered by Barker and the present author.

In a postscript, Pryce also refers to similar work carried out by Papapetrou. Pryce and others refer to the total energy $\mathrm{P}_{0}$ as $E$ and $\mathrm{P}_{0}$ is defined in (5). The total spin is $S$. To lowest order, $\mathrm{P}=\mathrm{mv}$ so that in the non-relativistic limit (4) becomes:

$$
\Delta \mathrm{r}=\frac{S \times v}{2 m c^{2}} .
$$

We notice that this is a lowest-order (or $c^{-2}$ ) contribution.

Pryce made extensive use of the energy-momentum tensor in his analysis and it is interesting to note that Møller also used this tensor extensively [16,17]. Møller pointed out that, in special relativity, 
a particle with structure and spin $S$ (its angular momentum vector in the rest system $K(0)$ ) must always have a finite extension and that there is a '...difference $\Delta \mathrm{r}$ between the simultaneous positions of the center of mass in its own rest system $\mathrm{K}(0)$ and system $\mathrm{K}$ (obtained from $\mathrm{K}(0)$ by a Lorentz transformation with velocity v)...' where:

$$
\Delta \mathrm{r}=\frac{S \times v}{m c^{2}}(\text { Moller })
$$

The factor of 2 difference between Equations (10) and (11) was explained in detail in [18] and is due to the fact that Møller's derivation did not include the fact that most physical systems include an extra Lorentz transformation arising from Thomas precession (rotation) caused by acceleration. On the other hand, the phenomenon is already incorporated in the work of Pryce, and it is interesting to note that it is in keeping with the localization postulate of Newton-Wigner since the $\Delta \mathrm{r}$ is associated with the uniform motion of the center of energy of the system.

At this stage, I recall how Barker and I arrived at identifying the NW position operator as the position operator in the classical two-body relativistic case and how the Møller approach entered the picture. We were interested in spin precession in gravitational physics and, motivated by the specific predictions of Schiff [19], we decided to thoroughly investigate the history of the area and come up with, hopefully, a new approach. This was achieved by our 1-body paper entitled "Derivation of the Equations of Motion of a Gyroscope from the Quantum Theory of Gravitation" [20], where, in contrast to previous approaches, the effects of spin were included ab initio. Our approach was based on a potential derived from Gupta's quantum theory of gravitation [21] for the scattering of two spin $\frac{1}{2}$ particles. Next, based on the universal nature of gravitation, we obtained the corresponding classical results by letting [20].

$$
\frac{1}{2} \hbar \sigma \rightarrow S
$$

Our resulting Hamiltonian immediately enabled us to obtain Schiff's results for 1-body spin precession but with different results for the orbital equations. Later, in [22], we showed that our results and these of Schiff were the same if one took account of the fact that apparent differences are due only to different locations of the center of mass. Such differences were explained to some extent by Møller's transformation, given by Equation (11). Still to be explained is why we obtained the same results as Schiff for spin precession since our results differed from these of Schiff by factor of the order of $\Delta \mathrm{r}$ given in Equation (4). The answer is that what is generally referred to as actual spin precession results are obtained after averaging over a complete orbit and, if we examine (11), we note that the change in $S$ over the orbit is also of order $S$ and negligible (since non-relativistic spin precession results are, by definition, linear in the spin). Also, the change in v over the orbit is also zero and hence the change is $\Delta \mathrm{r}$ over the orbit is zero. Of course, it should be emphasized that this will no longer be true for more relativistic motion (as, for example, the LIGO-VIRGO orbits).

The systems of primary interest are 2-body systems and the first calculation of a 2-body spin precession was carried out in [23] and we note that the theoretical prediction has been verified [10]. In fact, our calculation had classical position operators built in to it ab initio.

Next, we note that the different choices of the location of the center of mass goes back to the work of Mathisson [2], who pioneered the study of spin in general relativity. He defined the spin in the rest system as the 3-vector $S$, which he then generalized to the 4-vector $S_{\alpha}$, which he wrote in terms of the second rank term $S^{\alpha \beta}$, as discussed in Section 2. We note that Equation (1) has the same form as the Pauli-Lubanski vector but in the latter the spin tensor is replaced by the spin plus orbital momentum tensor and the position 4-vector is replaced by the momentum 4-vector to give a 4-vector on the left hand side whose scalar product is a Casimir invariant.

The relation between $S_{\alpha \beta}$ and $S^{\mu}$ is not unique $[18,22]$ but depends on the choice of the so-called spin supplementary condition, which in turn depends on the coordinate system chosen. Popular choices are $S^{\alpha \beta} U_{\beta}=0$ and $S^{\alpha \beta} p_{\beta}=0$ where $U_{\beta}$ and $p_{\beta}$ are 4 - velocity and 4 - momentum vectors. 
However, these choices, because they are covariant, are not suitable for treating an accelerating particle, and so, we introduced a new supplementary condition [3,22]:

$$
2 S^{i 4}+S^{i j} U_{j}=0
$$

This choice also got rid of problems (such as classical Zitterbewewegung [4], which we note is due to an interference between positive and negative components in the wave packet and this is eliminated by choosing a wave packet pertaining to a positive energy [8]) This non-covariant choice (13) corresponds to the choice of the N W positive operator $\Delta \mathrm{r}$ given by Equation (4) Thus, such a choice should by made in all spin calculations. To our knowledge, this is the first time that the Newton-Wigner and Pryce operator was applied to strictly a classical problem [20,21]. The reason why Møller's result does not directly correspond to the correct position operator is that it resulted in calculating the shift in the center-of-mass due to Lorentz transformations without rotations, thereby ignoring the rotation associated with the acceleration of the moving particle [23], as we have explained in [18]. Møller's choice, $S^{\beta \sigma} U_{\beta}=0$, corresponds to the choice of the rest frame of the electron (a non-inertial frame) and thus the quantum generalization does not include the Thomas contribution [23]. Our choice (essentially what Jackson [24] refers to as the non-rotating frame) actually corresponds to the choice of an inertial frame given by (13). In fact, a heuristic explanation of why the corrected Møller results gives the NW position operator is that it continually shifts the center-of-mass as time develops so that the wave function retains its localization property, in conformity with the basic requirement of Newton-Wigner.

It is also notable that the non-relativistic limit of the NW position operator (4) is the same as the FW operator if one drops the second and third terms in the Equation (23) of the FW paper. It was actually derived in [18] by generalizing Møller's classical result [18] to include both rotation and quantum effects and was used to derive all relativistic terms involving spin terms arising from the Dirac equation that were formerly derived using the more complex FW transformation.

\section{Discussion}

In summary, the NW-Pryce position operator (4), generally referred to simply as the NW position operator, which we initially identified in our two-body gravitational spin precession paper [24], is the operator of choice in all applications in QED [17] and classical general relativistic theory [22-24]. It also plays an essential role in the analysis of gravitational wave generation [23,25,26].

The recent observation of gravitational radiation from black hole and neutron star binary systems requires a sophisticated numerical analysis which must include the incorporation of a PNW position operator, as was realized particularly by Damour and collaborators [27].

Finally, we note that the above also provides the basis of an explanation of hidden momentum [2831], which is a relativistic quantity which occurs in otherwise non-relativistic systems in order to obtain the correct energy and momentum balances. Taking the time derivative of (6) and neglecting the very small second order terms, we find that:

$$
\Delta \mathrm{P}=\frac{S \times a}{2 c^{2}}
$$

where $\mathrm{a}$ is the acceleration, which is a very general expression for hidden momentum and in the case where $\mathrm{a}=\mathrm{e} \mathrm{E} / \mathrm{m}$, where $\mathrm{E}$ is the electric field and in particular, in electrodynamics, where the magnetic dipole momentum $\mathrm{M}$ is proportional to $\mathrm{S}$, we obtain [30]:

$$
\Delta \mathrm{P}=\mathrm{k} \frac{M \times E}{c^{2}},
$$

where $\mathrm{k}$ is a constant. This is a common result appearing in most discussions of hidden momentum in electromagnetism [28-31].

Funding: This research received no external funding. 
Acknowledgments: This research received no external funding. This work was partially supported by LSU Boyd Professor Research support funds. The author would like to thank Professor Pierre Ramond for introducing him to his own work [14] on position operators.

Conflicts of Interest: The author declares no conflict of interest.

\section{References}

1. O'Connell, R.F. Rotation and Spin in Physics. In General Relativity and John Archibald Wheeler, 1st ed.; Ciufolini, I., Matzner, R., Eds.; Springer: New York, NY, USA, 2010.

2. Mathisson, M. Neue mechanik materieller systems. Acta Phys. Pol. 1937, 6, 163.

3. Barker, B.M.; O'Connell, R.F. Nongeodesic motion in general relativity. Gen. Relat. Gravit. 1974, 5, 539. [CrossRef]

4. Foldy, L.; Wouthuysen, S.A. On the Dirac theory of spin $1 / 2$ particles and its non-relativistic limit. Phys. Rev. 1950, 78, 29. [CrossRef]

5. Bjorken, J.D.; Drell, S.D. Relativistic Quantum Mechanics; McGraw Hill: New York, NY, USA, 1964.

6. Schweber, S.S. An Introduction to Relativistic Quantum Field Theory; Harper and Row: New York, NY, USA, 1962.

7. Pryce, M.H.L. The mass-centre in the restricted theory of relativity and its connexion with the quantum theory of elementary particles. Proc. Roy. Soc. 1948, A195, 62.

8. Newton, T.D.; Wigner, E. Localized states for elementary systems Revs. Modern Phys. 1949, 21, 400. [CrossRef]

9. Lorente, M.; Roman, P. General expressions for the position and spin operators of relativistic systems. J. Math. Phys. 1973, 15, 70. [CrossRef]

10. Breton, R.P.; Kaspi, V.M.; Kramer, M.; McLaughlin, M.A.; Lyutikov, M.; Ransom, S.M.; Stairs, I.H.; Ferdman, R.D.; Camilo, F.; Possenti, A. Relativistic spin precession in the double pulsar. Science 2008, 321, 104. [CrossRef] [PubMed]

11. Hanson, A.J.; Regge, T. The relativistic spherical top. Ann Phys. 1974, 87, 498. [CrossRef]

12. Dirac, P.A.M. Forms of relativistic dynamics. Rev. Mod. Phys. 1949, 21, 392. [CrossRef]

13. Hojman, S.; Regge, T. Studies in Mathematical Physiocs, Essays in Honor of Valentine Bargmann; Lieb, E.H., Simon, B., Wightman, A.S., Hojman, S., Eds.; Princeton University Press: Princeton, NJ, USA, 1976.

14. Ramond, P. Group Theory. A Physicist's Survey; Cambridge University Press: Cambridge, UK, 2010.

15. O'Connell, R.F.; Wigner, E.P. On the relation between momentum and velocity for elementary systems. Phys. Lett. 1977, 61A, 353. [CrossRef]

16. Møller, C. On the Definition of the Centre of Gravity of an Arbitrary Closed System in the Theory of Relativity. Available online: https:/www.stp.dias.ie/Communications/DIAS-STP-Communications-005-Moller.pdf (accessed on 22 January 2020).

17. Møller, C. The Theory of Relativity, 2nd ed.; Oxford University Press: Oxford, UK, 1972.

18. O'Connell, R.F. Electron interaction with the spin angular momentum of the electromagnetic field. J. Phys. A 2017, 50, 085306. [CrossRef]

19. Schiff, L.I. Motion of a gyroscope according to Einstein's theory of gravitation. Proc. Natl. Acad. Sci. USA 1960, 46, 871. [CrossRef]

20. Barker, B.M.; O'Connell, R.F. Derivation of the equations of motion of a gyroscope from the quantum theory of gravitation. Phys. Rev. D 1970, 2, 1428. [CrossRef]

21. Barker, B.M.; Gupta, S.N.; Haracz, R.D. One-graviton exchange interaction of elementary particles. Phys. Rev. 1966, 149, 1027. [CrossRef]

22. Barker, B.M.; O'Connell, R.F. General Relativistic Effects in Binary Systems. Available online: https: //ui.adsabs.harvard.edu/abs/1978pans.proc..437B/abstract (accessed on 22 January 2020).

23. Barker, B.M.; O'Connell, R.F. Gravitational two-body problem with arbitrary masses, spins, and quadrupole moments. Phys. Rev. D 1975, 12, 329. [CrossRef]

24. Jackson, J.D. Classical Electrodynamics, 3rd ed.; Wiley: New York, NY, USA, 1998.

25. Kidder, L.E. Coalescing binary systems of compact objects to (post)5/2-Newtonian order. V. Spin effects. Phys. Rev. D 1995, 52, 821. [CrossRef] [PubMed] 
26. O'Connell, R.F. Gravito-Magnetism in one-body and two-body systems: Theory and Experiment, Invited Lecture. In "Atom Optics and Space Physics", Proc. of Course CLXVIII of the International School of Physics "Enrico Fermi", Varenna, Italy, 2007; Arimondo, E., Ertmer, W., Schleich, W., Eds.; IOS: Amsterdam, The Netherlands, 2009.

27. Damour, T.; Jaranowski, P.; Schafer, G. Hamiltonian of two spinning compact bodies with next-to-leading order gravitational spin-orbit coupling. Phys. Rev. D 2008, 77, 064032. [CrossRef]

28. Griffiths, D.J. Resource letter EM-1: Electromagnetic momentum. Am. J. Phys. 2012, 80, 7. [CrossRef]

29. Barnett, S.M. Comment on "Trouble with the Lorentz law of force: Incompatibility with special relativity and momentum conservation". Phys. Rev. Lett. 2013, 110, 089402. [CrossRef] [PubMed]

30. O'Connell, R.F. Equations of Motion in Relativistic Gravity; Springer Fundamental Theories of Physics; Puetzfeld, D., Ed.; Springer: Berlin, Germany, 2015.

31. Boyer, T.H. Concerning "hidden momentum". Am. J. Phys. 2008, 76, 190. [CrossRef]

(C) 2020 by the author. Licensee MDPI, Basel, Switzerland. This article is an open access article distributed under the terms and conditions of the Creative Commons Attribution (CC BY) license (http://creativecommons.org/licenses/by/4.0/). 